\title{
The Screening of Rubella Virus, Cytomegalovirus, Hepatitis B Virus, and Toxoplasma gondii Antibodies in Prepregnancy and Reproductive-Age Women in Tabriz, Iran
}

\author{
Edris Nabizadeh $\left(\mathbb{D},{ }^{1}\right.$ Anahita Ghotaslou $\left(\mathbb{D},{ }^{2}\right.$ Behnaz Salahi $\left(\mathbb{D},{ }^{3}\right.$ and Reza Ghotaslou $(\mathbb{D})^{1}$ \\ ${ }^{1}$ Infectious and Tropical Diseases Research Center, Tabriz University of Medical Sciences, Tabriz, Iran \\ ${ }^{2}$ Student Research Center, Medipol University, Ankara, Turkey \\ ${ }^{3}$ Razi Hospital, Tabriz University of Medical Sciences, Tabriz, Iran
}

Correspondence should be addressed to Reza Ghotaslou; rzgottaslo@yahoo.com

Received 27 October 2021; Accepted 27 December 2021; Published 10 January 2022

Academic Editor: Atif Amin Baig

Copyright (c) 2022 Edris Nabizadeh et al. This is an open access article distributed under the Creative Commons Attribution License, which permits unrestricted use, distribution, and reproduction in any medium, provided the original work is properly cited.

\begin{abstract}
Objectives. The organisms of Toxoplasma gondii, Rubella virus, Cytomegalovirus, and Herpes simplex virus as an acronym of TORCH are major pathogens in prepregnancy and reproductive-age women. These microorganisms are considered a serious problem and cause $2-3 \%$ of all birth defects in the fetus. Our study was aimed at screening the seroprevalence of TORCH antibodies among prepregnancy and reproductive-age women in Tabriz, Iran. Design and Setting. This study was carried out in 2726 prepregnancy and reproductive-age women, who were referred to the laboratory for prenatal TORCH screening. To detect the presence of IgG, IgM antibodies and Hepatitis B surface antigen against these microorganisms were carried out using a chemiluminescence immunoassay analyzer (CLIA). Results. In the current study, the rates of anti-Toxoplasma gondii IgG, anti-Rubella virus IgG, and anti-Cytomegalovirus IgG were found in 722 cases (26.5\%), 2579 cases (94.6\%0), and 2718 cases (99.7\%), respectively. Moreover, the rates of anti-Toxoplasma gondii IgM, anti-Rubella virus IgM, and antiCytomegalovirus IgM were discovered in 10 cases $(0.4 \%), 13$ cases $(0.5 \%)$, and 16 cases $(0.6 \%)$, respectively. The Hepatitis B surface antigen was found in 32 cases $(1.2 \%)$. The dissemination of positive TORCH in various ages was different $(P<0.05)$. Conclusions. In our study, the seroprevalence of acute TORCH infections was relatively low. Due to the probability of vertical transmission to the fetus during pregnancy and the unpleasant complication of these pathogens, it is essential to be screened for detection of specific IgG and IgM antibodies in reproductive ages.
\end{abstract}

\section{Introduction}

The infections of Toxoplasma gondii (TOX), Rubella virus (RUV), Cytomegalovirus (CMV), and Herpes simplex virus (HSV), as an acronym of TORCH, are major pathogens with serious problems in prepregnancy and reproductive-age women. They are considered to cause $2-3 \%$ of all birth defects in the fetus. Moreover, the letter "o" of TORCH as an abbreviation that refers to others can include some pathogen agents such as Arbovirus B19, Hepatitis B virus (HBV), Varicella-zoster virus, human immunodeficiency virus, and Zika virus $[1,2]$. The seroprevalence of these infections is different in various geographical areas [3]. Disease caused by TOX is generally with no sign and with mild infection in healthy individuals, and it may lead to serious problems in the fetus, which result in stillbirth, blindness, and mental retardation [4]. Rubella appears as a simple disease with mild symptoms and usually no specific complication. Despite these findings, whenever it occurs in a prepregnancy and reproductive-age woman, it can cause death and abortion [5]. CMV is one of the most common viral agents causing infection in utero, and its prevalence has been estimated at $0.3-2.4 \%$ of all successful deliveries. Infection due to CMV can be associated with severe complications that can eventually lead to growth retardation, jaundice, hepatosplenomegaly, and intracerebral damage $[6,7]$. Viral hepatitis caused 
TABLE 1: The seroprevalence of TORCH IgG and IgM among 2726 women who categorized based on age.

\begin{tabular}{|c|c|c|c|c|c|c|c|c|c|c|c|c|c|c|}
\hline \multirow{4}{*}{$\begin{array}{l}\text { Pathogen } \\
\text { Age group } \\
14-24 \text { y (653 cases) }\end{array}$} & \multicolumn{4}{|c|}{ TOX } & \multicolumn{4}{|c|}{ RUV } & \multicolumn{4}{|c|}{$\mathrm{CMV}$} & \multirow{2}{*}{\multicolumn{2}{|c|}{$\begin{array}{c}\text { HBV } \\
\text { HBsAg }\end{array}$}} \\
\hline & \multicolumn{2}{|c|}{$\operatorname{IgG}$} & \multicolumn{2}{|c|}{$\operatorname{IgM}$} & \multicolumn{2}{|c|}{ IgG } & \multicolumn{2}{|c|}{$\operatorname{IgM}$} & \multicolumn{2}{|c|}{ IgG } & \multicolumn{2}{|c|}{$\operatorname{IgM}$} & & \\
\hline & $\mathrm{P}$ & $\mathrm{N}$ & $\mathrm{P}$ & $\mathrm{N}$ & $\mathrm{P}$ & $\mathrm{N}$ & $\mathrm{P}$ & $\mathrm{N}$ & $\mathrm{P}$ & $\mathrm{N}$ & $\mathrm{P}$ & $\mathrm{N}$ & $\mathrm{P}$ & $\mathrm{N}$ \\
\hline & 109 & 544 & 0 & 653 & 545 & 108 & 2 & 651 & 648 & 5 & 6 & 647 & 1 & 652 \\
\hline $25-35$ y (1361) & 353 & 1008 & 8 & 1353 & 1339 & 22 & 11 & 1350 & 1358 & 3 & 10 & 1351 & 16 & 1345 \\
\hline $36-45$ y $(712)$ & 260 & 452 & 2 & 712 & 695 & 17 & 0 & 712 & 712 & 0 & 0 & 712 & 15 & 697 \\
\hline
\end{tabular}

by some viruses such as Hepatitis $B$ virus is a common form of the disease worldwide. Chronic hepatitis patients can transmit the virus for years without any symptoms. Prepregnancy and reproductive-age mothers are believed to be the most important risk factor for transmitting the virus to the fetus. Nowadays, to screen HBV infections in prepregnancy and reproductive-age women, the HBsAg test is recommended [8]. It is a normal initial detection of the TORCH pathogen which is important for treatment and prevention strategies for adverse fetal outcomes [9]. The characteristic of these pathogens is that they are asymptomatic at the beginning of the infection, and they cannot be diagnosed based on their clinical features. The tracing of the IgM antibody against TORCH pathogens is the best aspect of the recognition of these infections [10]. This study was aimed at screening the seroprevalence of TORCH infections among prepregnancy and reproductive-age women in Tabriz.

\section{Materials and Methods}

2.1. The Patients. This study was carried out on 2726 prepregnancy and reproductive-age women, who were referred to the laboratory for prenatal screening in Tabriz, Iran, from 2018 to 2020. Awareness consent was received from all women who were referred to screening for TORCH. Blood samples were collected from each person and were centrifuged at $1200 \times \mathrm{g}$ for $15 \mathrm{~min}$ to separate the serum from the blood.

2.2. The Screening. To detect the presence of anti-CMV, TOX, RUV IgG, and IgM antibodies and also HBsAg, the serologic testing was carried out using a chemiluminescent immunoassay (Liaison ${ }^{\circledR} \mathrm{XL}$, Italy). According to the manufacturer's instructions (Liaison Company, Italy), the analysis of the test results was carried out based on the cut-off activity index. Additionally, the quality control process of the device and kits was performed according to the relevant standards.

2.3. Statistical Analysis. Statistical analysis was carried out by SPSS 26 (SPSS Inc., Chicago, Illinois, US).

\section{Results}

To determine the seroprevalence of TORCH, this study was conducted in 2726 cases of reproductive-aged women. The mean age of women was $30 \pm 7$ years old (range 14-45). As shown in Table 1, all women based on age were divided into three categories, and the seroprevalence of TORCH was determined in each category. The distribution of positive
TABLE 2: The seroprevalence of tested TORCH pathogens among 2726 women.

\begin{tabular}{lcccc}
\hline \multirow{2}{*}{ Pathogens } & \multicolumn{2}{c}{ IgG } & \multicolumn{2}{c}{ IgM } \\
& Positive & Negative & Positive & Negative \\
\hline TOX & $722(26.5 \%)$ & $2004(73.5 \%)$ & $10(0.4 \%)$ & $2716(99.6 \%)$ \\
RUV & $2579(94.6 \%)$ & $147(5.4 \%)$ & $13(0.5 \%)$ & $2713(99.5 \%)$ \\
CMV & $2718(99.7 \%)$ & $8(0.3 \%)$ & $16(0.6 \%)$ & $2710(99.4 \%)$ \\
\hline
\end{tabular}

TORCH in various ages was different $(P<0.05)$. The rate of anti-TOX IgG antibody, anti-Rubella IgG, and antiCMV IgG were found in 722 cases (26.5\%), 2579 cases (94.6\%), and 2718 cases (99.7\%), respectively. Moreover, the rate of anti-TOX IgM antibody, anti-Rubella IgM, and anti-CMV IgM was discovered in 10 cases (0.4\%), 13 cases $(0.5 \%)$, and 16 cases $(0.6 \%)$, respectively. The seroprevalence of HBsAg Hepatitis $B$ virus (HBsAg positive) as other pathogen-related to TORCH was in $32(1.2 \%)$ cases. The seroprevalence of tested TORCH pathogens among 2726 women is shown in Table 2.

\section{Discussion}

Intrauterine infections related to $\mathrm{TORCH}$ pathogens can lead to serious fetal complications, despite most of these organisms not being associated with a serious injury in the mothers and appearing often as a mild conflict. Conventional screening via serological assays is effective in the detection of TORCH infections, before pregnancy, prepregnancy, and reproductive-age women. In this investigation, we had analyzed the seroprevalence of TORCH agents (TOX, RUV, CMV, and Hepatitis B virus) among prepregnancy and reproductive-age women in Tabriz, Iran. This issue is helpful for the understanding of the diverse TORCH agents spreading in this area. The strength of our research may be a high sample size over a short study period of two years, and it was conducted for the first time in this geographical area. The weakness of our work may be the lack of full patient's follow-up, which will be pursued in the future and lack of definitive confirmation of positive cases by amniocentesis sampling. Due to various factors, the exact spread of TORCH infections is still unknown in most parts of the world [2]. In the present study, acute infections due to TOX, CMV, and RUV were $0.4 \%, 0.5 \%$, and $0.6 \%$, respectively. Toxoplasma gondii is an intracellular pathogen that is transmitted in a variety of ways, including consuming uncooked meat, contact with some animals especially cats, and contaminated foodstuffs or water. Disease caused by this 
agent can lead to serious problems in the fetus $[11,12]$. In the current study, the positive cases were detected for antiTOX IgG (26.5\%) and IgM (0.4\%). These findings were different from the average found in previous studies in Iran that reported $35-41 \%$. The reason for this discrepancy may be due to low sample size in the previous study [13]. In addition, the seroprevalence of TOX infections is different in various countries, for example, UK 9.1-7.7\% [14], India $28 \%$ [12], and Canada 59.8\% [15]. This difference in the frequency is likely due to the nutritional habits (consuming well-cooked food and consumption of frozen meat), socioeconomic state, geographical difference, and improved level of health in meat production [16]. Rubella appears as a simple viral disease with mild symptoms or is even asymptomatic in neonates and not common in adults [17]. On the other hand, the prevalence of RUV infections in developed countries has been few due to vaccination programs. Previous studies have reported the seroprevalence of RUV in prepregnancy and reproductive-age women in several countries ranging from 83.4 to $97.9 \%$, for example, India (83.4\%) [18], southern Italy (85.8\%) [19], Turkey (96.3\%) [20], and Nigeria $(97.9 \%$ ) [21]. The results of our study revealed that $89.4 \%$ of the women had anti-RUV IgG antibodies and also $0.5 \%$ had Rubella IgM. These results are almost in agreement with other studies' findings in some parts of Iran, which suggest the success of the vaccination program [22]. RUV disease usually occurs in cases that come from countries without a vaccination program against this virus or people who refuse to be vaccinated.

CMV is one of the most common viral agents classified in the herpes family. This pathogen can be associated with severe complications that can eventually lead to growth retardation, jaundice, hepatosplenomegaly, and intracerebral damage. The virus is also found globally in developed and underdeveloped countries, especially in areas with a high economic crisis. Its seroprevalence is estimated between $45 \%$ and $100 \%$ and 0.2 $2.2 \%$ morbidity [23]. The current study indicated that the anti-CMV IgG antibody in $99.7 \%$ cases, as well as $0.6 \%$ of women, had a positive IgM antibody. In a study from Iran carried out in 2015, the prevalence of anti-CMV-IgG and IgM antibodies during the first trimester of pregnancy was reported as $98.8 \%$ and $5 \%$, respectively [22]. Besides, in the previous investigations, the seropositive rates of CMV IgG in prepregnancy and reproductive-age women in the various counties were revealed to be different, for example, $84.5-95 \%$ in Turkey, $84 \%$ in Spain, 56.8 in Australia, and 39-94.7\% in the USA [24]. One of the important public health issues worldwide is $\mathrm{HBV}$ infection. According to some reports, an estimated 325 million persons in the world were infected with this virus. HBV is a contagious infection that can affect neonates via a vertical transmission [25]. The universal seroprevalence of $H B V$ infection is varied and classified based on the seroprevalence in four categories including $\geq 8 \%$ which indicates a high level of infection, 5$7.9 \%$ high-intermediate, $2-4.9 \%$ low-intermediate, and $<2 \%$ low [26]. Studies on the prevalence of Hepatitis $B$ in prepregnancy and reproductive-age women in some countries are as follows: USA $0.38 \%$, in comparison with foreign women who were born in Asia 2-8.7\% [27] and Ghana 7.7\% [28]. In the current study, the seroprevalence of HBsAg was $1.2 \%$. Our finding is equal to the result (1.21\%) of the study carried out by Shoghli et al., which was performed in seven provinces of Iran [29]. Also, the seropositivity rate of HBsAg in our study was higher than in different cities of Iran, including Babol (0.18\%), Dehloran (0.59), and Amol (0.62\%) [29, 30]. Moreover, the finding of our study almost was comparable with a survey conducted by Tanriverdi et al., in Turkish prepregnancy and reproductive-age women, in our neighbor country $(1.2 \%)$ [31], which probably refers to the hypothesis of ethnic diversity and genetics as a risk factor [32]. But, further studies are needed to prove this hypothesis. Considering TORCH as major pathogens in prepregnancy and reproductive-age women, which is the cause of $2-3 \%$ of all birth defects in the fetus and serious problems in the fetus, the screening of TORCH is important. Since these diseases are asymptomatic in a prepregnancy and reproductive-age woman and can be transmitted to the fetus, its normal, initial recognition is vital for the prevention and treatment of diseases due to TORCH. On the other hand, molecular tests are not fully available to them; therefore, we rely on serology testing. On the other hand, the prevalence of TORCH infection varies in different regions, and the prevalence may be high in other parts of the country, so conducting such studies for epidemiological purposes is important. In our study, the seroprevalence of acute TORCH infections was relatively low. However, due to the vertical transmission to the fetus during pregnancy and the unpleasant complication of these pathogens, it is essential to take all necessary precautions, and screening in women must be done at reproductive ages.

\section{Conclusion}

In our study, the seroprevalence of acute TORCH infections was relatively low. Due to the probability of vertical transmission to the fetus during pregnancy and the unpleasant complication of these pathogens, it is essential to be screened by detection of specific IgG and IgM antibodies in reproductive ages.

\section{Data Availability}

The data used to support the findings of this study are included in the article.

\section{Conflicts of Interest}

The authors declare that they have no conflicts of interest.

\section{Authors' Contributions}

Reza Ghotaslou conceived and designed the study; Edris Nabizadeh, Anahita Ghotaslou, and Reza Ghotaslou initiated the comprehensive research and analyzed the data; Anahita Ghotaslou, Behnaz Salahi, Edris Nabizadeh, and Reza Ghotaslou wrote and revised the paper. All authors read and approved the manuscript. 


\section{Acknowledgments}

We would like to thank the Tabriz University of Medical Sciences, Faculty of Medicine, and the Central Laboratory of Tabriz and all those who helped us in this study.

\section{References}

[1] Z. Li, C. Yan, P. Liu, R. Yan, and Z. Feng, "Prevalence of serum antibodies to TORCH among women before pregnancy or in the early period of pregnancy in Beijing," Clinica Chimica Acta, vol. 403, no. 1-2, pp. 212-215, 2009.

[2] Y. Qi, S. Zhu, C. Li et al., "Seroepidemiology of TORCH antibodies in the reproductive-aged women in China," European Journal of Obstetrics \& Gynecology and Reproductive Biology, vol. 254, pp. 114-118, 2020.

[3] N. Kumari, N. Morris, and R. Dutta, "Is screening of TORCH worthwhile in women with bad obstetric history: an observation from eastern Nepal," Journal of Health, Population, and Nutrition, vol. 29, no. 1, p. 77, 2011.

[4] R. Gilbert and C. Dezateux, "Newborn screening for congenital toxoplasmosis: feasible, but benefits are not established," Archives of Disease in Childhood, vol. 91, no. 8, pp. 629-631, 2006.

[5] A. Nardone, A. Tischer, N. Andrews et al., "Comparison of rubella seroepidemiology in 17 countries: progress towards international disease control targets," Bulletin of the World Health Organization, vol. 86, no. 2, pp. 118-125, 2008.

[6] A. Kenneson and M. J. Cannon, "Review and meta-analysis of the epidemiology of congenital cytomegalovirus (CMV) infection," Reviews in Medical Virology, vol. 17, no. 4, pp. 253-276, 2007.

[7] T. Vilibic-Cavlek, S. Ljubin-Sternak, M. Ban, B. Kolaric, M. Sviben, and G. Mlinaric-Galinovic, "Seroprevalence of TORCH infections in women of childbearing age in Croatia," The Journal of Maternal-Fetal \& Neonatal Medicine, vol. 24, no. 2, pp. 280-283, 2011.

[8] M. Silasi, I. Cardenas, J. Y. Kwon, K. Racicot, P. Aldo, and G. Mor, "Viral infections during pregnancy," American Journal of Reproductive Immunology, vol. 73, no. 3, pp. 199-213, 2015.

[9] L.-C. Wang, F. Yan, J. X. Ruan, Y. Xiao, and Y. Yu, “TORCH screening used appropriately in China?- Three years results from a teaching hospital in Northwest China," BMC Pregnancy and Childbirth, vol. 19, no. 1, p. 484, 2019.

[10] M. Sen, B. Shukla, and B. Tuhina, "Prevalence of serum antibodies to TORCH infection in and around Varanasi, northern India," Journal of Clinical and Diagnostic Research: JCDR, vol. 6, no. 9, pp. 1483-1485, 2012.

[11] G. Pappas, N. Roussos, and M. E. Falagas, "Toxoplasmosis snapshots: global status of Toxoplasma gondii seroprevalence and implications for pregnancy and congenital toxoplasmosis," International Journal for Parasitology, vol. 39, no. 12, pp. 1385-1394, 2009.

[12] K. R. Prasoona, B. Srinadh, T. Sunitha et al., "Seroprevalence and influence of torch infections in high risk pregnant women: a large study from South India," The journal of Obstetrics and Gynecology of India, vol. 65, no. 5, pp. 301-309, 2015.

[13] A. Daryani, S. Sarvi, M. Aarabi et al., "Seroprevalence of Toxoplasma gondii in the Iranian general population: a systematic review and meta-analysis," Acta Tropica, vol. 137, pp. 185194, 2014.
[14] J. Q. Nash, S. Chissel, J. Jones, F. Warburton, and N. Q. Verlander, "Risk factors for toxoplasmosis in pregnant women in Kent, United Kingdom," Epidemiology \& Infection, vol. 133, no. 3, pp. 475-483, 2005.

[15] V. Messier, B. Lévesque, J. F. Proulx et al., "Seroprevalence of toxoplasma gondii among Nunavik Inuit (Canada)," Zoonoses and Public Health, vol. 56, no. 4, pp. 188-197, 2009.

[16] A. Hofhuis, W. van Pelt, Y. T. H. P. van Duynhoven et al., "Decreased prevalence and age-specific risk factors forToxoplasma gondiilgG antibodies in the Netherlands between 1995/1996 and 2006/2007," Epidemiology \& Infection, vol. 139, no. 4, pp. 530-538, 2011.

[17] K. Kaur and A. Oberoi, "Prevalence of various torch infections among females of reproductive age group," Journal of Evolution of Medical and Dental Sciences, vol. 4, no. 65, pp. 11391-11396, 2015.

[18] D. E. Muliyil, P. Singh, S. K. Jois et al., "Sero-prevalence of rubella among pregnant women in India, 2017," Vaccine, vol. 36, no. 52, pp. 7909-7912, 2018.

[19] S. Calimeri, A. Capua, V. la Fauci, R. Squeri, O. C. Grillo, and D. L. Giudice, "Prevalence of serum anti-rubella virus antibodies among pregnant women in southern Italy," International Journal of Gynecology \& Obstetrics, vol. 116, no. 3, pp. 211-213, 2012.

[20] E. Ç. Tanriverdi, L. Dikbaş, A. L. Handan, B. G. Kadioğlu, and Z. Özkurt, "Seroprevalance of rubella antibodies among pregnant women in a regional maternity hospital in eastern Turkey," Mediterranean Journal of Infection Microbes and Antimicrobials, vol. 7, 2018.

[21] M.-D. Amina, S. Oladapo, S. Habib, O. Adebola, K. Bimbo, and A. Daniel, "Prevalence of rubella IgG antibodies among pregnant women in Zaria, Nigeria," International health, vol. 2, no. 2, pp. 156-159, 2010.

[22] R. Moniri, F. Baghbani Taheri, S. Sadat, and Z. Heidarzadeh, "Prevalence of serum antibodies to TORCH infection in the first trimester of the pregnancy in Kashan," Iranian Journal of Neonatology IJN, vol. 6, no. 1, pp. 8-12, 2015.

[23] M. Kumar, M. B. Nizam, and M. Mugunthan, "Seroprevalence of cytomegalovirus infection in antenatal women in a tertiary care center in western India," Journal of Marine Medical Society, vol. 19, no. 1, p. 51, 2017.

[24] G. Shrivastava, G. Bhatambare, and K. Patel, "Seroprevalance of toxoplasma, rubella, CMV and HSV infection in pregnant women in central India," International Journal of Health System and Disaster Management, vol. 2, no. 3, p. 166, 2014.

[25] M. T. Frempong, P. Ntiamoah, M. E. Annani-Akollor et al., "Hepatitis B and C infections in HIV-1 and non-HIV infected pregnant women in the Brong-Ahafo region, Ghana," PLoS One, vol. 14, no. 7, article e0219922, 2019.

[26] Organization, WH, Global hepatitis report 2017, World Health Organization, 2017.

[27] T. Y. Walker, E. A. Smith, N. Fenlon et al., "Characteristics of pregnant women with hepatitis B virus infection in 5 US public health jurisdictions, 2008-2012," Public Health Reports, vol. 131, no. 5, pp. 685-694, 2016.

[28] B. A. Dortey, E. A. Anaba, A. T. Lassey, N. K. R. Damale, and E. T. Maya, "Seroprevalence of hepatitis B virus infection and associated factors among pregnant women at Korle-Bu Teaching Hospital, Ghana," PLoS One, vol. 15, no. 4, article e0232208, 2020. 
[29] A. Shoghli, S. M. Nabavi, S. M. Alavian et al., "Hepatitis B surface antigen prevalence in pregnant women: a cross-sectional survey in Iran," International Journal of Preventive Medicine, vol. 5, Suppl 3, pp. S213-S218, 2014.

[30] L. Kheiri and S. Makvandi, "The prevalence of hepatitis B surface antigen (HBsAg) and its influencing factors in pregnant women referring to healthcare centers of Dehloran, Iran in 2011-2012," Journal of Midwifery and Reproductive Health, vol. 3, no. 3, pp. 424-429, 2015.

[31] E. Ç. Tanrıverdi, Z. Özkurt, B. G. Kadıŏlu et al., "Seroprevalence of hepatitis $\mathrm{B}$, hepatitis $\mathrm{C}$, and HIV in pregnant women from eastern Turkey," The Turkish Journal of Gastroenterology, vol. 30, no. 3, pp. 260-265, 2019.

[32] N. Akbar, B. Basuki, Mulyanto, D. H. Garabrant, A. Sulaiman, and H. M. Noer, "Ethnicity, socioeconomic status, transfusions and risk of hepatitis B and hepatitis C infection," Journal of Gastroenterology and Hepatology, vol. 12, no. 11, pp. 752757, 1997. 